\title{
Redes Oportunísticas de Comunicação Segura para Monitoramento de Riscos de Sinistros
}

\author{
Lucas S. dos Santos ${ }^{1}$, Victor C. Messner ${ }^{1}$, \\ Diego L. C. Dutra ${ }^{1}$ e Claudio L. Amorim ${ }^{1}$ \\ ${ }^{1}$ Universidade Federal do Rio de Janeiro \\ Rio de Janeiro - RJ - Brasil \\ $\{$ lseveriano, vcmessner, ddutra, amorim\}@cos.ufrj.br
}

\begin{abstract}
We present a cost-effective solution for applications of monitoring of accident risks based on an interest-centric opportunistic secure-communication network, namely RadNet. The monitoring system provides regular collection of events and its transmission to a system of control of accident risks and Forensic Audit. To this end, event transmission requires Confidentiality, Integrity, and Availability, a triad that RadNet can satisfy by mitigating the vulnerabilities of using IP addresses and end-to-end communication.
\end{abstract}

Resumo. Apresentamos uma solução custo-efetiva para aplicações de monitoramento de riscos de sinistros baseada em uma rede oportunística de comunicação segura centrada em interesses, denominada RadNet. O sistema de monitoramento provê a coleta regular de eventos e sua transmissão para um sistema de controle de riscos de sinistros e Auditoria Forense. Para este fim, a transmissão de eventos exige Confidencialidade, Integridade e Disponibilidade, tríade que a RadNet consegue satisfazer ao mitigar as vulnerabilidades de utilização de endereços IP e comunicação fim-a-fim.

\section{Introdução}

A Internet das Coisas - Internet of Things (IoT) - é um cenário da Tecnologia da Informação e Comunicação (TIC), no qual um grande número de dispositivos (ou nós) interconectados em rede podem gerar dados abertos para consumo por terceiros [Zanella et al. 2014]. Notavelmente, uma rede IoT oferece um extraordinário potencial de alavancar novos serviços de TIC ao gerar e compartilhar dados de informação, porém introduz um grande desafio aos projetistas de sistemas IoT: garantir a segurança dos dados propagados em uma rede de capilaridade variada com dispositivos heterogêneos de capacidades computacional e de transmissão limitadas [Sedjelmaci et al. 2017].

Neste trabalho, consideramos um ambiente de IoT de comunicação sem fio composto principalmente por dispositivos Raspberry Pi e celulares, opcionalmente com suporte de nó(s) infraestruturado(s) (i.e., computador-servidor convencional). Neste ambiente, a segurança dos dados é definida como a aderência a tríade: Confidencialidade, Integridade e Disponibilidade. Uma questão complexa inerente ao projeto de um ambiente IoT, que impacta diretamente à sua segurança, é a sua diversidade de áreas de aplicações, que inclui transporte público, monitoramento de riscos à saúde das pessoas, automação industrial e resposta de emergência a catástrofes [Al-Fuqaha et al. 2015]. Nessas áreas, as decisões devem ser tomadas com base em informações de sensoriamento local dos 
ambientes, tornando imprescindível garantir a integridade dos dados coletados e também prover mecanismos criptográficos para proteger sua confidencialidade, se necessário.

A implementação de técnicas de segurança na transmissão da informação pressupõe a instalação de componentes ativos e passivos na rede IoT: i) sensores (e.x., gases e temperatura) e sistemas de extinção de chamas para ambientes físicos; e ii) firewalls e sistemas de detecção/prevenção de intrusões para infraestruturas de comunicação virtuais. Independentemente do ambiente sendo protegido, a técnica de segurança utilizada deve garantir a Auditoria Forense dos eventos coletados, o que torna os repositórios de eventos um componente crítico do projeto de gerência de segurança da informação. Neste escopo, os repositórios são alvos valiosos para usuários maliciosos, exigindo assim soluções inovadoras custo-efetivas para proteção e manutenção da integridade da informação armazenada, tal como a solução RadNet-S [Lima et al. 2018, Dutra et al. 2012a] eficientemente aplicada no suporte à gerência de segurança da informação.

A principal contribuição deste trabalho é propor um modelo de Monitoramento de Riscos de Sinistros (MRS) usando uma rede oportunística de comunicação segura centrada em interesses, que explora os benefícios operacionais e custo-efetivos do RadNet-S orientando-o para sistemas MRS e proteção local de ambientes. O restante deste trabalho está organizado da seguinte forma: a Seção 2 descreve o protocolo RadNet para redes oportunísticas, abordando seus conceitos fundamentais. A Seção 3 descreve a aplicação MRS. Na Seção 4, apresentamos as conclusões e trabalhos futuros.

\section{Protocolo RadNet}

O protocolo de rede oportunística centrada em interesses RadNet foi originalmente proposto para uso em redes de comunicação MANETS (Mobile Ad-hoc NETworkS) [Dutra et al. 2012a] com pouca ou nenhuma infraestrutura. Na RadNet, em vez de endereçamento IP fim-a-fim, as aplicações enviam mensagens cujos cabeçalhos usam prefixos ativos (PAs). Um PA possui dois componentes: um prefixo e um nome de interesse, construídos pelo nó e aplicação local, respectivamente. A ausência de infraestrutura pré-estabelecida de rede é compensada pelo suporte ao tratamento das duas funções dos PAs que cada nó implementa na camada de rede: encaminhamento e entrega de mensagens.

Nesse sentido, o prefixo é construído de modo a permitir o encaminhamento probabilístico de mensagens e, ainda, servir de identificador do nó gerador e ser utilizado para endereçamento pelas aplicações. Complementarmente, a aplicação atribui um nome de interesse para identificar o conteúdo que ela irá compartilhar [Dutra et al. 2012a]. A Figura 1 apresenta os componentes do PA (a) cabeçalho da mensagem (b) na RadNet.

\subsection{Comunicação centrada em interesse}

O exemplo da Figura 2 ilustra a transmissão de dados entre quatro dispositivos na RadNet, onde o raio de transmissão sem fio é delimitado pela circunferência tracejada. Nesse cenário, cada nó contém um PA com dois campos numéricos, e um interesse registrado na camada de rede. A comunicação inicia através do envio da mensagem do nó A com prefixo [1;5] e interesse [Futebol], i.e., PA:[1;5;Futebol]. O nó B, no raio de alcance da transmissão de A, recebe o pacote proveniente de A, e encaminha a mensagem de A por 


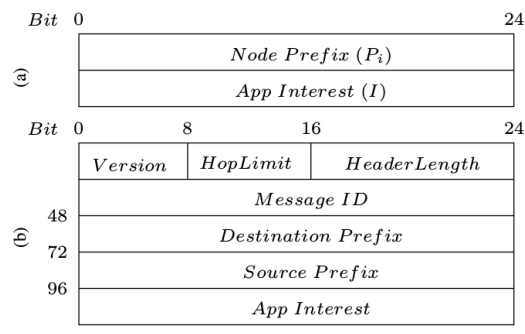

Figura 1. Elementos da RadNet: (a)Prefixo Ativo e (b) Cabeçalho da mensagem Extraído de [Dutra et al. 2012a]

haver casamento de prefixos de A com B (ex. critério de casamento: ambos PAs tem o mesmo valor 5 no $2^{\circ}$ campo). O nó A recebe o pacote de volta encaminhado por $\mathrm{B}$, porém detecta que o pacote já foi processado anteriormente e o descarta. O Nó C, recebe e encaminha a mensagem de A por haver casamento de prefixos no $1^{\circ}$ campo(=1). O nó $\mathrm{D}$, ao receber o pacote, detecta que possui o mesmo interesse (Futebol) e o repassa para a aplicação local, mas não há casamento de prefixos, logo descarta a mensagem.

É importante notar a ausência da localização (endereço IP) do nó destinatário na mensagem. Ainda, o uso de PA também permite esconder a localização do nó origem. Complementarmente, o interesse e o conteúdo da mensagem podem ser criptografados para garantir a comunicação segura entre nós, usando o suporte à comunicação por interesse implementado na camada de rede de cada nó. Desta forma, a RadNet provê mecanismos para garantir a confidencialidade e a integridade de mensagens.

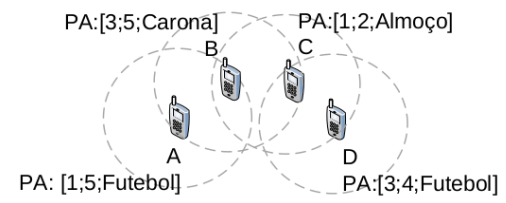

Figura 2. Exemplo de comunicação usando Radnet - Extraído de [Salles 2014]

\section{Aplicação: monitoramento de riscos de sinistros}

Partindo das experiências de trabalhos anteriores usando RadNets [Rodriguez et al. 2017, Gonçalves et al. 2016, Dutra et al. 2015, Dutra et al. 2012b, Moraes et al. 2012, Granja et al. 2010], projetamos um sistema de monitoramento de sinistros usando uma rede oportunística RadNet como o meio de transmissão de mensagens. Entre as vantagens desta abordagem, incluímos a escalabilidade do sistema, a redundância e a sua utilização em ambientes sem infraestrutura prévia. A Figura 3 apresenta a aplicação de sistema de monitoramento, no qual o dispositivo Raspberry, acoplado com sensores, coleta informações, registra eventos de sensoriamento e os envia para o servidor central responsável por manter o controle e registro de eventos recebidos dos dispositivos que compõem a RadNet. Além disso, em caso de detecção de algum risco serão enviados alarmes para agentes responsáveis credenciados que estejam 
na região coberta pela RadNet. Os dados coletados pelos sensores são disseminados pela rede através do protocolo de comunicação RadNet, sem a necessidade de uma infraestrutura prévia de rede. Porém, em caso de alarmes, estes também poderão ser enviados para funcionários credenciados e localizados remotamente, através de gateway entre a RadNet e uma rede de comunicação infraestruturada (IP).

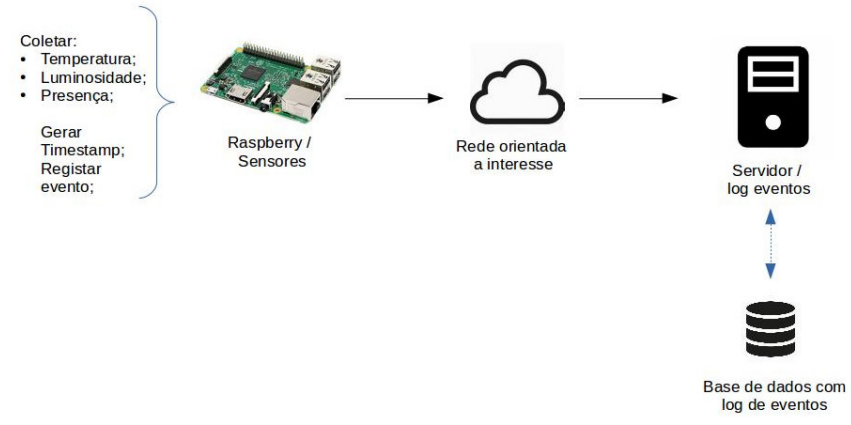

Figura 3. Representação em alto nível de aplicação de monitoramento

O servidor contém uma base de dados para armazenamento e observação dos eventos gerados pelo sensoriamento realizados pelos dispositivos dispersos formadores da rede. Através desta base de dados é possível relacionar os dispositivos na rede com as informações coletadas e os eventos gerados por eles. Desta forma, o sistema fornece meios para armazenar, gerenciar e visualizar o histórico de eventos para fins de auditoria forense. Com relação à proteção dos dados trafegados, o projeto do sistema de monitoramento de sinistros aqui descrito prevê o uso de soluções de criptografia como as descritas em [Bashirpour et al. 2018].

\subsection{Estudo de caso: MonitoraCT}

Para ilustrar melhor a nossa proposta, apresentamos um cenário no qual o sistema MRS deverá detectar os riscos de sinistros no subsolo do Centro de Tecnologia/UFRJ localizado na Ilha do Fundão. Os sinistros incluem incêndio, vazamento de gás tóxico e inundação. Esse sistema MRS é baseado em imagens coletadas e mensagens de alerta geradas pela aplicação MRS distribuída utilizando uma rede de dispositivos sem fio instalada no referido subsolo entre a Brigada de Incêndio(BI) e o Laboratório de Instrumentação Fotônica (LIF). As Figuras 4, 5 apresentam as localizações da BI e do LIF. As circunferências nas figuras delimitam o alcance do rádio dos dispositivos da rede.

A RadNet irá enviar de forma segura (criptografada) as informações fornecidas pelos sensores, regularmente ou quando requisitadas, para serem processadas pela aplicação de controle e exibidas no computador de monitoramento instalado na BI. Outras pessoas autorizadas pela administração do CT, em trânsito na região, também terão acesso a essas informações através de seus celulares.

\section{Considerações Finais e Trabalhos Futuros}




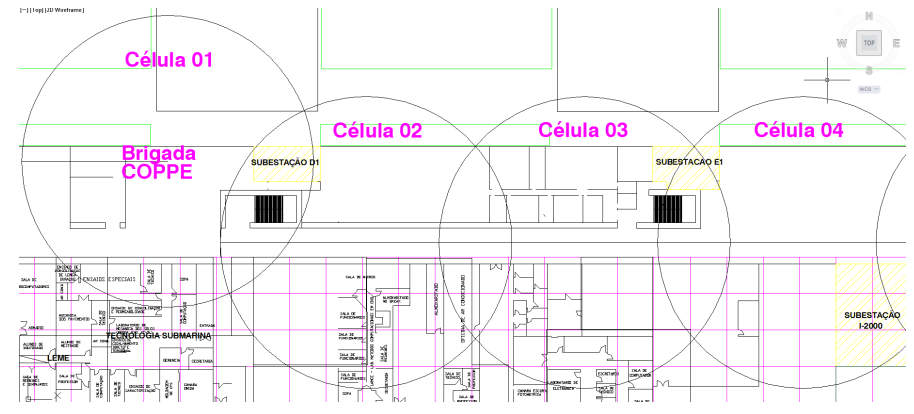

Figura 4. Localização da brigada de incêndio no Subsolo do Bloco I (Fonte: Escritório do Planejamento - Acesso em 15 de abril de 2014)

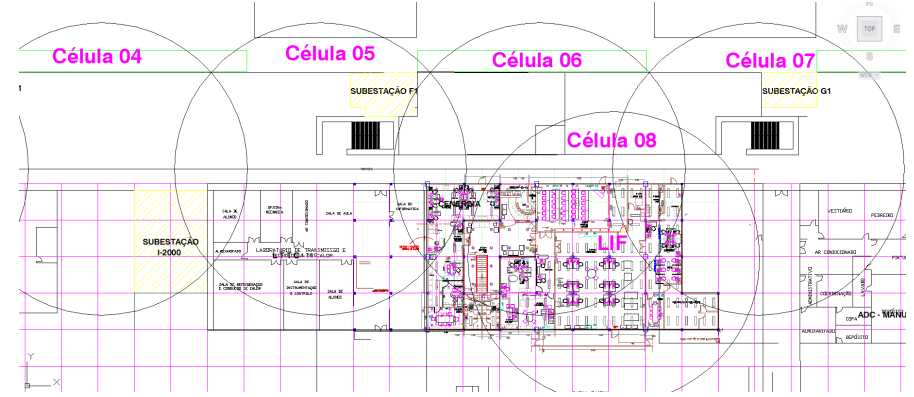

Figura 5. - Localização do LIF no Subsolo do Bloco I (Fonte: Escritório do Planejamento - Acesso em 15 de abril de 2014)

Este trabalho apresentou um proposta de sistema de monitoramento de sinistros utilizando redes oportunísticas RadNets, sendo descrito como esse tipo de solução se adéqua aos cenários de IoT. Como perspectiva de trabalhos futuros, pretende-se desenvolver a aplicação de monitoramento proposta neste trabalho e implementá-la em ambientes similares ao descrito no estudo de caso. Por fim, o planejamento do ambiente da RadNet prevê o uso da técnica de ajuste de potência de dispositivos de comunicação sem fio descrita em [Amorim and Kostin 2018].

\section{Referências}

Al-Fuqaha, A., Guizani, M., Mohammadi, M., Aledhari, M., and Ayyash, M. (2015). Internet of things: A survey on enabling technologies, protocols, and applications. IEEE Communications Surveys \& Tutorials, 17(4):2347-2376.

Amorim, C. L. and Kostin, S. (2018). Método de ajuste de potência de equipamentos de transmissão sem fio segundo o perfil de obstáculos e ocupação do terreno e seu uso. INPI: Carta Patente No PI 0400115-0.

Bashirpour, H., Bashirpour, S., Shamshirband, S., and Chronopoulos, A. T. (2018). An improved digital signature protocol to multi-user broadcast authentication based on elliptic curve cryptography in wireless sensor networks (wsns). Mathematical and Computational Applications, 23(2).

Dutra, D. L. C., Moraes, H. F., and Amorim, C. L. (2015). Radflow: An interest-centric task based dataflow runtime. In 2015 International Symposium on Computer Architecture and High Performance Computing Workshop (SBAC-PADW), pages 115-119. 
Dutra, R. C., Moraes, H. F., and Amorim, C. L. (2012a). Interest-centric mobile ad hoc networks. In Network Computing and Applications (NCA), 2012 11th IEEE International Symposium on, pages 130-138. IEEE.

Dutra, R. C., Moraes, H. F., and Amorim, C. L. (2012b). Interest-centric mobile ad hoc networks. In 2012 IEEE 11th International Symposium on Network Computing and Applications, pages 130-138.

Gonçalves, F. B., França, F. M. G., and de Amorim, C. L. (2016). Interest-centric vehicular ad hoc network. In 2016 IEEE 12th International Conference on Wireless and Mobile Computing, Networking and Communications (WiMob), pages 1-10.

Granja, R. S., Dutra, R. C., de Moraes, H. F., and de Amorim, C. L. (2010). Samcra: Um sistema para avaliação experimental de redes ad hoc. In Seção de Ferramentas XXVIII SBRC.

Lima, L., Cabral Filho, P., Dutra, D. L., Amorim, C. L., Macedo, E. L. C., Silva, R. S., Coutinho, M. A., and de Moraes, L. F. M. (2018). Radnet-s: Um mecanismo para transmissão segura e secreta de registros syslog. In XXIII Workshop de Gerência e Operação de Redes e Serviços (WGRS).

Moraes, H. F., Benitez, N. R. P., Dutra, R. C., and Amorim, C. L. (2012). On developing interest-centric applications for ad hoc networks. In 2012 IEEE International Symposium on a World of Wireless, Mobile and Multimedia Networks (WoWMoM), pages $1-3$.

Rodriguez, L. G. A., de Jeus, J. A., do Rosário, V. M., da Silva, A. F., Peres, L. P., de Moraes, H. F., and de Amorim, C. L. (2017). mybee: An information system for precision beekeeping. In Proceedings of the 19th International Conference on Enterprise Information Systems - Volume 2: ICEIS, pages 577-587. INSTICC, SciTePress.

Salles, R. C. (2014). Avaliação de capacidade e consumo de energia de rede móvel ad hoc centrada em interesse. Master's thesis, Universidade Federal do Rio de Janeiro. https://www.cos.ufrj.br/index.php/pt-BR/publicacoes-pesquisa/details/15/2497.

Sedjelmaci, H., Senouci, S. M., and Taleb, T. (2017). An accurate security game for lowresource iot devices. IEEE Transactions on Vehicular Technology, 66(10):9381-9393.

Zanella, A., Bui, N., Castellani, A., Vangelista, L., and Zorzi, M. (2014). Internet of things for smart cities. IEEE Internet of Things Journal, 1(1):22-32. 